\title{
A National Survey of the Social and Emotional Differences Reported by Adults with Disability in Ireland Compared to the General Population
}

\author{
Roy McConkey (D)
}

check for updates

Citation: McConkey, R. A National Survey of the Social and Emotional Differences Reported by Adults with Disability in Ireland Compared to the General Population. Disabilities 2021, 1, 89-97. https://doi.org/10.3390/ disabilities1020008

Academic Editor: Reinie Cordier

Received: 3 February 2021

Accepted: 1 April 2021

Published: 7 April 2021

Publisher's Note: MDPI stays neutral with regard to jurisdictional claims in published maps and institutional affiliations.

Copyright: (C) 2021 by the author. Licensee MDPI, Basel, Switzerland. This article is an open access article distributed under the terms and conditions of the Creative Commons Attribution (CC BY) license (https:/ / creativecommons.org/licenses/by/ $4.0 /)$.
Institute of Nursing and Health Research, Ulster University, Newtownabbey BT37 0QB, UK; r.mcconkey@ulster.ac.uk

\begin{abstract}
The disadvantages experienced by adult persons with disabilities are well documented. However, limited evidence is available on the extent of differences in comparison with the nondisabled population. In this study, selected indicators of social status and mental wellbeing derived from past research, were used with national samples of adult persons in Ireland with a disability $(n=440)$ and without a disability $(n=880)$ recruited through household quota sampling. In addition, comparisons were drawn with equivalent data derived from a contemporaneous national census. Although many of the differences were statistically significant, the effect sizes were mostly medium to low. Moreover, when the inter-relationships among the various indicators was taken into account using Discriminant Analysis, persons with disabilities were less likely to be employed; they reported lower levels of social engagement and had poorer emotional wellbeing. They were also older, more likely to be single and have no children. The study illustrates the potential of using comparative data to monitor the impact of national actions taken to reduce the inequalities experienced by persons with disability as well as highlighting the arenas into which professional supports need to be focused.
\end{abstract}

Keywords: national survey; disability; adults; social inclusion; emotional wellbeing; Ireland; census; household survey

\section{Introduction}

The nations of the world have re-affirmed the rights of persons with disabilities to full and effective participation in society on an equal basis with their non-disabled peers [1]. Yet, these aspirations remain unfulfilled in most if not all countries, as a worldwide report of disability has documented [2]. Consequently, many governments have enacted legislation and policies aimed at reducing the inequalities and discrimination experienced by persons with disabilities - including those with chronic illnesses [3]. In Ireland, as in other countries, various high-level initiatives have been published which propose radical reforms in both the disability sector and mainstream provision [4]. Yet, these are often fragmented by sector such as education, employment and housing with little attention paid to identifying and responding to the priority inequalities experienced by persons with disability. Moreover, the effectiveness of these policy initiatives on promoting equality of opportunity are difficult to assess despite the recognition given in the UN Convention on the rights of persons with disabilities, of the need to collect statistical and research data (Article 31) [5].

An essential requirement in information gathering on this issue is a comparison between persons with and without disabilities. For such comparisons to be valid, a number of requirements have to be met [6]. Equivalent measures need to be taken with both groups at the same time and in ways that offset any communication difficulties on the part of persons with disabilities in particular. The samples should strive to be representative of both groupings as well as being matched in terms of other characteristics that may affect a disabled persons' equal participation in society such as gender and ethnicity [7]. Moreover, 
national samples are preferable due to possible regional variations such as urban-rural differences [8]. Together these requirements form a daunting challenge for researchers.

A national population census comes closest in terms of sampling requirements but only a limited number of measures can be collected with possibly a long time interval between each census. Nonetheless, census returns can provide useful comparative data on certain key indicators such as access to education and employment with the possibility of charting changes over longer periods of time.

A much less costly approach is the use of samples of the national population that allow for more detailed information to be gathered and on a more frequent basis. A common example is the use of household surveys [9]. However, this strategy seems to have been rarely deployed in the published literature relating to comparisons between people with and without disabilities, although a survey in the United Kingdom is a notable exception [10]. In part, this may be due to relatively small numbers of persons with disabilities identified in household surveys of the general population. Rather greater reliance has been paid to studies involving specially recruited samples only of people with disabilities and comparisons are based with pre-gathered data on non-disabled persons, albeit that other confounding factors are not easily controlled in such comparisons. For example, a pertinent review of studies relating to social relationships and mental wellbeing is available and which highlighted the increased risks experienced by people with physical disabilities [11].

The present study was undertaken in the Republic of Ireland which has a population of around 4.9 million. The purpose was to examine the feasibility of using household surveys to recruit quota samples of persons with and without disabilities so as to determine the differences between them on indicators of social and emotional wellbeing. A booster sample of persons with disabilities would be simultaneously recruited to increase their numbers for more robust statistical comparisons [12]. Comparisons could also be drawn with the limited data available from a national census that had been completed around the same time.

The study was undertaken in the context of a wider study into the perceived rights of persons with disabilities [13]. In order to reduce the interview burden on informants, a selection of indicators was deployed based on questions or scales that had been used or adapted from previous surveys. Nonetheless, samples of sufficient size would enable the inter-relationships among the indictors to be assessed using multi-variate analyses which small samples have not been able to do.

In summary, the aims of the study were as follows.

- To determine the value of using household surveys which incorporated a booster sample to contrast Irish persons with and without disabilities.

- To identify the social and emotional differences that most distinguish persons with a disability in the Irish population.

\section{Materials and Methods}

This study involved secondary analyses of social and emotional wellbeing measures of respondents who took part in a broader national survey undertaken in Ireland that was commissioned by the National Disability Authority (NDA) [14]. Comparisons are also drawn with publicly available information from the 2016 national census undertaken in Ireland [15].

The survey involved face-to-face, computer-aided interviews conducted at the homes of the respondents in early 2017. The survey questionnaires were developed in consultation with officials from the NDA and their advisers including the author, along with the staff of the market research company commissioned to undertake the data gathering. These were informed by reviews of past research $[10,16]$. 


\subsection{Interview Items}

In line with the aims of the study, two conceptual models informed the selection of items to assess respondents' social and emotional wellbeing. First, the ecological model of social inclusion proposed by Simplican et al. [17] which has two main dimensions-namely, social networks and community participation. Second, the model of personal wellbeing developed by UK Office of National Statistics [18]. The following scales and items were selected for use in the survey.

The Lubben Social Network Scale-6 [19] measures the size of the person's social network in terms of respondent's contact with friends and relatives and whether they could call on them for help and talk to them about private matters. The scores range from 6 to 30 with a high score indicative of having larger social networks. For the full sample in this survey, the Cronbach alpha for the scale was 0.882 .

Respondent's engagement in social activities was assessed by counting their participation in six exemplars as used in various studies [20]; including internet access, having a mobile phone, going on holiday, having a hobby and taking day trips. Scores ranged from 0 to 6 with high scores indicative of greater social engagement. The Cronbach alpha was 0.628 which is low but is probably reflective of the diversity of activities included.

Two items from the UK Office of National Statistics scale on personal wellbeing [21] relating to happiness and satisfaction with life were used on which respondents selected a score between 1 and 10. These two ratings were added to give a combined score that ranged from 2 to 20 with higher score indicative of having a happier and more satisfied life. The correlation between ratings on happiness and satisfaction was $r=0.891$.

Three items relating to mental wellbeing from the EU Quality of Life survey [22] were asked: "felt tense; felt lonely; felt down-hearted and depressed". Respondents responded on a six-point Likert scale ranging from "all of the time to none of the time". Scores ranged from 3 to 18 with higher scores reflective of better emotional wellbeing. The Cronbach alpha was 0.894 .

Demographic information about respondents was also collected (see Table 2). In addition, some of the demographic details could be compared to those reported in the 2016 national census that also identified persons with disability and long-lasting health conditions that were similar to those used in the survey. Comparisons between persons with and without disabilities could be ascertained from the census although no statistical tests could be undertaken as only grouped data are publicly available.

\subsection{Sampling}

The study involved a national sample of 1021 respondents plus a booster sample of an additional 273 people with disabilities. In all, 125 sampling locations across Ireland were selected randomly, based on a national list of District Electoral Divisions. Households in each location were further randomly selected. Only one person per household was eligible to participate in the study. Telephone contact identified the chosen individuals based on quotas applied for region, gender, age and social economic group with a tight geographical stratification to ensure that the findings would be representative of Irish adults aged $18+$. The booster sample of persons with disabilities was similarly chosen with regional quota controls to ensure it was in line with the number of people with disabilities in the main sample.

Trained interviewers visited the persons home and conducted face-to-face interviews with the chosen individual, but just over $7.3 \%(n=95)$ responded to the survey using a proxy. Of these, just over half $(56.8 \%, n=54)$ reported having a disability.

Fuller methodological details along with the interview schedule are available in the report published by the National Disability Authority [14].

For the purpose of this study, respondents were grouped into those with a self-declared disability $(n=440)$ and those who had not reported having a disability $(n=884)$. 


\subsection{Ethics}

In common with standard market research practice, formal ethical approval was not sought for the survey, but it was conducted under the code of conduct of the Association of Irish Market Research Organisations [23]. Respondents were informed verbally and in writing of the purposes of the survey and how the data would be used and shared. They were assured that their answers would be confidential and that no one would be identified in any reports. They could decline to answer any questions or to terminate the interview without giving a reason. Contact details were provided if respondents wished to comment or complain about the survey.

Anonymised data files were used for the secondary data analysis which were publicly available from the Irish Social Science Data Archive. Available at https://www.ucd.ie/ issda/ (accessed on 16 January 2021). The census data are also publicly available at https: / / data.cso.ie/\# (accessed on 18 January 2021).

\subsection{Data Analysis}

SPSS (version 25) (IBM, Armonk, NY, USA) was used for statistical analysis. Descriptive statistics illustrated the demographic characteristic of respondents with and without disabilities. Chi Sq tests were used to test for differences alongside an estimate of effect sizes (Cohen's W): values greater than 0.3 are indicative of a medium effect and over 0.5 for a strong effect. For continuous variables, comparisons between the two groups were made using T-Tests with an estimate made of effects sizes using Cohen's $\mathrm{d}$ : with values greater than 0.5 indicative of a medium effect size [24]. Throughout the probability level was set at $p<0.01$ given the multiple comparisons that were made.

A discriminant analysis was used to identify the social and emotional variables that most distinguished respondents with and without disabilities. This analysis was repeated for persons aged under 60 years to reduce possible effects of age-related, acquired disabilities.

\section{Results}

\subsection{Disabilities}

Within the survey, respondents were asked to identify if they had any of the longlasting conditions from a list presented to them. Overall, 440 respondents selected at least one condition and Table 1 summarises the number and percentage selecting each one. Over three quarters $(76 \%)$ reported one condition with $17 \%$ having two conditions and $7 \%$ three or more conditions.

Table 1. The number and percentage of respondents selecting each condition $(n=1294)$.

\begin{tabular}{cc}
\hline Condition & Number (\%) \\
\hline Physical Limitations & $246(19.0 \%)$ \\
Chronic Illness & $161(12.4 \%)$ \\
Hearing Loss & $53(4.1 \%)$ \\
Mental Health Difficulty & $53(4.1 \%)$ \\
Intellectual Disability & $51(3.9 \%)$ \\
Vision Difficulties & $39(3.0 \%)$ \\
Autism/ASD & $25(1.9 \%)$ \\
Other & $1(0.1 \%)$ \\
\hline
\end{tabular}

\subsection{Demographics}

Table 2 presents the demographic characteristics of respondents with and without disabilities along with the statistical tests of significance that were undertaken. Additionally, shown in square brackets is the comparable proportions of persons from the national census undertaken in 2016 on the same characteristics. However, the publicly available data for the census define adults as aged 15 years and over, whereas the survey population was aged 
18 years and over. This difference should be considered when comparing the characteristics of the survey population with the national census data.

Table 2. The demographic characteristics of survey respondents with and without disabilities. The equivalent proportions from the 2016 national census are shown in square brackets.

\begin{tabular}{|c|c|c|c|}
\hline & $\begin{array}{l}\text { Non-Disabled } \\
\quad(n=884)\end{array}$ & Disabled $(n=440)$ & $\begin{array}{c}\text { Statistical Difference } \\
\text { between the Two } \\
\text { Survey Groups }\end{array}$ \\
\hline \multicolumn{4}{|c|}{ Gender } \\
\hline Male & $417(48.8 \%)[49.3 \%]$ & $211(48.0 \%)[46.4 \%]$ & Chi Sq $=0.089$ \\
\hline Female & $437(51.2 \%)[50.7 \%]$ & $229(52.0 \%)[53.6 \%]$ & Not significant * \\
\hline \multicolumn{4}{|c|}{ Age Bands } \\
\hline 18-39 years & $381(44.6 \%)[45.2 \%]$ & $94(21.4 \%)[21.7 \%]$ & Chi Sq 110.79; \\
\hline $40-59$ years & $320(37.5 \%)[35.3 \%]$ & $158(35.9 \%)[29.7 \%]$ & $p<0.001$ \\
\hline $60+$ years & $153(17.9 \%)[19.5 \%]$ & $188(42.7 \%)[48.6 \%]$ & Cohen's W $=0.293$ \\
\hline \multicolumn{4}{|c|}{ Marital status } \\
\hline Married/Partner & $552(64.6 \%)[53.6 \%]$ & $205(46.6 \%)[43.1 \%]$ & Chi Sq 38.95; $p<0.001$ \\
\hline Single/Widowed/Divorced & $302(35.4 \%)[46.4 \%]$ & $235(53.4 \%)[56.9 \%]$ & Cohen's $W=0.174$ \\
\hline \multicolumn{4}{|c|}{ Social Class } \\
\hline $\mathrm{ABC} 1$ & $413(48.4 \%)[45.6 \%]$ & $145(33.0 \%)[35.0 \%]$ & Chi Sq 28.10; $p<0.001$ \\
\hline $\mathrm{C} 2 \mathrm{DEF}^{\#}$ & $441(51.6 \%)[54.5 \%]$ & $295(67.0 \%)[65.0 \%]$ & Cohen's $W=0.147$ \\
\hline \multicolumn{4}{|c|}{ Education } \\
\hline Primary & $40(4.7 \%)[9.8 \%]$ & $73(16.6 \%)[31.3 \%]$ & \\
\hline Secondary & $434(50.8 \%)[44.7 \%]$ & $254(57.7 \%)[44.7 \%]$ & Chi Sq 76.73; $p<0.001$ \\
\hline Third Level & $380(44.5 \%)[45.6 \%]$ & $113(25.7 \%)[24.0 \%]$ & Cohen's W = 0.244 \\
\hline \multicolumn{4}{|c|}{ Employment Status } \\
\hline Employed & $556(65.1 \%)[53.4 \%]$ & $125(28.4 \%)[22.3 \%]$ & Chi Sq 164.27; \\
\hline Not Employed & $185(21.7 \%)[27.4 \%]$ & $163(37.0 \%)[23.2 \%]$ & $p<0.001$ \\
\hline Retired & $113(13.2 \%)[19.1 \%]$ & $152(34.5 \%)[54.6 \%]$ & Cohen's W = 0.356 \\
\hline \multicolumn{4}{|c|}{ Children } \\
\hline None & $286(33.5 \%)$ & $200(45.5 \%)$ & Chi Sq 17.73; $p<0.001$ \\
\hline Yes & $568(66.5 \%)$ & $240(54.4 \%)$ & Cohen's $W=0.117$ \\
\hline \multicolumn{4}{|c|}{ Location } \\
\hline Urban & $553(64.8 \%)$ & $292(66.4 \%)$ & Chi Sq 0.332 \\
\hline Rural & $301(35.2 \%)$ & $148(33.6 \%)$ & Not significant * \\
\hline \multicolumn{4}{|c|}{ Nationality } \\
\hline Irish & $762(89.2 \%)$ & $408(92.7 \%)$ & Chi Sq 4.11 \\
\hline Non-Irish & $92(10.8 \%)$ & $32(7.3 \%)$ & Not significant * \\
\hline
\end{tabular}

As Table 2 summarises, the differences between the two groups are most apparent when the effect size is considered: the larger the effect size, the more significant is the difference between the two groups on that variable. Thus, persons with disabilities were less likely to be in employment, they are more likely to be aged 60 years and over, they are less likely to have attended third level education, more likely to be single, widowed or divorced, to be of lower socio-economic status and less likely to have children.

A similar pattern can also be discerned in the data from the national census (within the margin of error of $\pm 3 \%$ ) with the exception of marital and employment status. The greater variation possibly arose from the difference in ages recorded for adults which was 15 years and over in the Census and 18 years and over in the household survey. Nonetheless the differences identified in the survey are replicated in the census data which adds further validity to these distinguishing characteristics of persons with disability.

By contrast, there were no differences between persons with and without disabilities in terms of gender, location and nationality nor by region of the country (although the latter is not shown in Table 2). 


\subsection{Social and Emotional Wellbeing}

The mean scores and standard deviations on the four measures of social and emotional wellbeing included in the survey are shown in Table 3.

Table 3. The mean scores and standard deviations on the four measures of social and emotional wellbeing in the two groups of respondents with and without disabilities.

\begin{tabular}{cccc}
\hline Measure & $\begin{array}{c}\text { Non-Disabled } \\
(\boldsymbol{n}=\mathbf{8 8 4})\end{array}$ & $\begin{array}{c}\text { Disabled } \\
(\boldsymbol{n = 4 4 0 )}\end{array}$ & Statistical Difference \\
\hline Social Networks & $18.85(4.94)$ & $17.77(5.03)$ & $\begin{array}{c}\mathrm{F}=13.60 ; p<0.001 \\
\text { Cohen's d }=0.217\end{array}$ \\
Social Engagement & $4.48(1.34)$ & $3.46(1.61)$ & $\begin{array}{c}\mathrm{F}=146.7 ; p<0.001 \\
\text { Cohen's d }=0.689\end{array}$ \\
Happiness and Satisfaction & $16.34(2.74)$ & $14.84(3.73)$ & $\begin{array}{c}\mathrm{F}=67.23 ; p<0.001 \\
\text { Cohen's d }=0.458\end{array}$ \\
Emotional Wellbeing & $16.24(2.18)$ & $14.47(3.78)$ & $\begin{array}{c}\mathrm{F}=110.0 ; p<0.001 \\
\text { Cohen's d }=0.574\end{array}$ \\
\hline
\end{tabular}

Although all differences were statistically significant, in terms of effect size, the difference between the two groups of respondents was most evident in their social engagement activities, with people with disabilities having less engagement. Similarly, people with disabilities rated themselves as having poorer emotional wellbeing and lower happiness and satisfaction with life. Although persons with disabilities had lower scores in terms of support from friends, the effect size was less strong.

\subsection{Discriminant Analysis}

The variables that that had proved significant in the above analyses were then entered into a discriminant analyses to identify the variables that most discriminated the two groupings of respondents with and without disabilities. This analysis also took account of the inter-relationships among the variables. Due to missing data (3.9\% of cases), the sample size was reduced to 1244 . The resulting model was significant and consisted of one function (Canonical correlation 0.499; Wilk's Lambda 0.751; Chi Sq 354.5; df 6; $p<0.001$ ). The Discriminant analysis was repeated with respondents aged under 60 years of age to reduce the effects of acquired disabilities in old age (Canonical correlation 0.462; Wilk's Lambda 0.787; Chi Sq 217.4; df 6; $p<0.001$ ). Table 4 summarises the structure matrix of the variables that contributed significantly to with the discriminant function in the two models. These values shown are similar to the variable loadings in a factor analysis.

Table 4. The structure matrix of variables that best discriminated respondents with and without disabilities for all ages and for persons under 60 years of age.

\begin{tabular}{ccc}
\hline Variables & All Ages $(n=\mathbf{1 2 4 4})$ & Under 60 Years $(n=\mathbf{9 1 1})$ \\
\hline Employed & 0.638 & 0.634 \\
Social Engagement & 0.604 & 0.509 \\
Age of Person & 0.543 & 0.302 \\
Emotional Wellbeing & 0.517 & 0.624 \\
Marital Status & 0.307 & 0.274 \\
Having Children & 0.184 & 0.283 \\
\hline
\end{tabular}

The variables that most distinguished respondents with disabilities of all ages from those without disabilities was their non-employed status, lack of social engagement, being older, poorer emotional wellbeing and more likely not to be married or to have children. It is the combination of all these variables that most distinguished persons with disability in this Irish sample. 
For persons under 60 years, the same six variables contributed to the discriminant function but the weight of the loadings varied somewhat with poorer emotional wellbeing and having children loading higher and age less so.

However, in both models, the other variables that had distinguished the groups in the bi-variate analyses as shown in Tables 2 and 3, did not add significantly to the discriminant. They were level of education, social networks, feeling happy and satisfied, and social class. This suggests that the indicators included in the model better capture the major impacts of disability on the lives of Irish individuals.

\section{Discussion}

This study is unique in several ways. Information was obtained through face-toface interviews with individual respondents who were quota sampled across Ireland to represent population characteristics but who lived in districts and households that were randomly sampled. Comparisons with national census data broadly confirmed this. The social and emotional corollaries of disability and chronic illness in adulthood were examined. These are often overlooked when the focus is on medical or therapeutic assessments and interventions. A range of disabling conditions and age groups were represented in the survey which suggests a commonality of impact across different conditions, although further comparative research would be needed to confirm this.

The study suggests that the lack of gainful employment is the most potent discriminator of people with disabilities in Ireland. Rather they are more likely to be unemployed, judged unable to work or retired from working and therefore are likely to be dependent on State-provided social security payments. Indeed, repeated studies have reported the disadvantages faced by people with disabilities in terms of employment [25] but this study highlights its pre-eminent social impact on their lives. Interestingly the type of work people undertook as reflected in their socio-economic classification was not a strong predictor of difference. Crudely stated, this implies that any work is better than none.

Irish people with disabilities experienced other forms of societal exclusion as captured in the measure of social engagement. This added to the discriminant function implying that lack of work and social engagement presented a double jeopardy for persons with disabilities. Previous research has suggested that transport issues and lack of disposable income may be barriers to engaging in leisure activities and using technology rather than a disability per se $[16,26]$.

The findings also highlight the poorer emotional wellbeing among person with disabilities, especially for those aged under 60 years of age. Whether or not this is a cause or an effect of exclusion from employment or social engagement is debatable, but this finding points to the need for greater attention to be paid to the social and emotional consequences of disability which are often overlooked in professional services geared to their physical and medical needs [11].

Unsurprisingly, age emerged as a further discriminating characteristic: disabilities increase as people age, especially when they are over 60 years old but also with those aged 40 years and over. However, age was not the most discriminating characteristic especially for those under 60 years of age. Rather, these findings suggest that the impact of ageing could be mitigated by employment creation, enhanced social engagement and better emotional well-being; all of which are amenable to change, even among older persons.

Marital status and having children were other significant discriminators of Irish persons with and without disabilities although to a lesser extent than those already discussed. These are further evidence for the social impact of disabilities although they are not inevitable or necessarily the choice of individuals. Unlike previous studies, the social networks of persons with disabilities in Ireland were broadly similar to those of their nondisabled peers and were not a significant discriminator when other possible co-variates were taken into account. This would imply that disabled persons had similar opportunities to form deeper relationships. However, others have suggested that persons with disabilities are discouraged from having intimate relationships on which marriage, partnerships and 
pro-creation are built [27]. These more subtle forms of discrimination and exclusion need to be challenged.

The study yielded some unexpected results in terms of indicators that failed to discriminate Irish persons with and without disabilities despite the findings from previous studies (though it must be stressed that this was because other related variables had been taken into account). These included: lower levels of education [28] (which seems to be of less importance in adult life although of vital importance to children and young adults with disabilities); a higher incidence of disabling condition in rural areas [8] (this issue may be less important in a relatively small country such as Ireland); less happiness and life satisfaction [29] (poorer emotional wellbeing seemed to be a better predictor of mental health) and smaller social networks [11] (this may be more specific to Irish culture that places a high emphasis on social connections). That said, these indicators may be important to include in future surveys in other countries but with the caution that the inter-relationships among variables should also be considered.

Finally, the present survey had some limitations, notably the sample was biased against respondents with only primary education which the quota sampling failed to rectify. Although quota sampling has its limitations, random sampling of individuals requires much larger numbers with higher associated costs. The inclusion of more detailed social indicators and measures of emotional wellbeing would provide deeper insights into the differences experienced by people with disabilities. Additionally, the contribution that different impairment conditions could make to social and emotional discrepancies could not be determined due to the small numbers of respondents with differing conditions but obtaining comparable representative samples for different conditions would be especially challenging.

\section{Conclusions}

The study confirmed the value of household surveys with booster samples in recruiting broadly representative samples on which comparisons can be drawn between persons with and without disabilities. It identified indicators that future surveys should include, especially in relation to employment, social exclusion and mental health. This would augment information available from a national census or, ideally, such indicators could be included in future censuses. Moreover, government policies and actions in support of persons with disabilities need to promote their access to gainful work, increase social engagement and address emotional wellbeing. Repeating the survey at regular intervals would help to monitor the implementation of the UN Convention on the rights of persons with disabilities and of national policies to equalize opportunities for persons with disabilities. The core question being, are the disparities between persons with and without disabilities decreasing?

Funding: This research was funded by the National Disability Authority (NDA) of Ireland.

Institutional Review Board Statement: Ethical review and approval were waived for this study, as it involved the secondary analysis of anonymized information that was publicly available.

Informed Consent Statement: Informed consent was obtained from all participants involved in the study.

Data Availability Statement: The data used in this study are available from: Irish Social Science Data Archive. Available at: https:/ / www.ucd.ie/issda/ (accessed on 16 January 2021).

Acknowledgments: The data gathering was undertaken by Behaviour and Attitudes, Dublin on commission from the National Disability Authority (NDA).

Conflicts of Interest: The author declares no conflict of interest. 


\section{References}

1. United Nations. Convention Rights of Persons with Disabilities; United Nations: Geneva, Switzerland, 2006.

2. World Health Organization; World Bank. World Report on Disability; WHO: Geneva, Switzerland, 2011.

3. Groce, N.; Trani, J. Disability and the Millennium Development Goals; United Nations Publication: Geneva, Switzerland, 2011.

4. Linehan, C.; O’Doherty, S.; Tatlow-Golden, M.; Craig, S.; Kerr, M.; Lynch, C.; McConkey, R.; Staines, A. Mapping the National Disability Policy Landscape; School of Social Work, Trinity College Dublin: Dublin, Ireland, 2014.

5. Lang, R.; Kett, M.; Groce, N.; Trani, J.F. Implementing the United Nations Convention on the rights of persons with disabilities: Principles, implications, practice and limitations. Alter Eur. J. Disabil. Res. 2011, 5, 206-220. [CrossRef]

6. Bryman, A. Social Research Methods, 4th ed.; Oxford University Press: Oxford, UK, 2012.

7. Kavanagh, A.M.; Krnjacki, L.; Aitken, Z.; LaMontagne, A.D.; Beer, A.; Baker, E.; Bentley, R. Intersections between disability, type of impairment, gender and socio-economic disadvantage in a nationally representative sample of 33,101 working-aged Australians. Disabil. Health J. 2015, 8, 191-199. [CrossRef] [PubMed]

8. Zhao, G.; Okoro, C.A.; Hsia, J.; Garvin, W.S.; Town, M. Prevalence of disability and disability types by urban-rural county classification-US, 2016. Am. J. Prev. Med. 2019, 57, 749-756. [CrossRef] [PubMed]

9. Deaton, A. The Analysis of Household Surveys: A Microeconometric Approach to Development Policy; The World Bank: Washington, DC, USA, 2018.

10. Office of National Statistics. Life Opportunities Survey. Available online: https://www.gov.uk/government/collections/lifeopportunities-survey (accessed on 3 February 2021).

11. Tough, H.; Siegrist, J.; Fekete, C. Social relationships, mental health and wellbeing in physical disability: A systematic review. BMC Public Health 2017, 17, 1-8.

12. Smith, P. Booster Samples of Marginal Groups vs. Separate Focussed Studies; University of Southampton: Southhampton, UK, 2019.

13. McConkey, R. Public perceptions of the rights of persons with disability: National surveys in the Republic of Ireland. Alter Eur. J. Disabil. Res. 2020, 14, 128-139. [CrossRef]

14. National Disability Authority. National Survey of Public Attitudes to Disability in Ireland 2017; NDA: Dublin, Ireland, 2017.

15. Central Statistics Office, Republic of Ireland. Databases. Available online: https://www.cso.ie/en/databases/ (accessed on 3 February 2021).

16. Hästbacka, E.; Nygård, M.; Nyqvist, F. Barriers and facilitators to societal participation people with disabilities: A scoping review of studies concerning European countries. Alter Eur. J. Disabil. Res. 2016, 10, 201-220. [CrossRef]

17. Simplican, S.C.; Leader, G.; Kosciulek, J.; Leahy, M. Defining social inclusion of people with intellectual and developmental disabilities: An ecological model of social networks and community participation. Res. Dev. Disabil. 2015, 38, 18-29. [CrossRef] [PubMed]

18. Randall, C.; Cochrane, A.; Jones, R.; Manclossi, S. Measuring National Well-Being in the UK: International Comparisons. 2019. Office for National Statistics. Available online: https:/ / www.ons.gov.uk/peoplepopulationandcommunity/wellbeing/articles/ measuringnationalwellbeing/internationalcomparisons2019 (accessed on 3 February 2021).

19. Lubben, J.; Blozik, E.; Gillmann, G.; Iliffe, S.; von Renteln Kruse, W.; Beck, J.C.; Stuck, A.E. Performance of an abbreviated version of the Lubben Social Network Scale among three European community dwelling older adult populations. Gerontologist 2006, 46, 503-513. [CrossRef] [PubMed]

20. Cordier, R.; Milbourn, B.; Martin, R.; Buchanan, A.; Chung, D.; Speyer, R. A systematic review evaluating the psychometric properties of measures of social inclusion. PLoS ONE 2017, 12, e0179109. [CrossRef] [PubMed]

21. Office of National Statistics. Surveys Using Our Four Personal Well-Being Questions. Available online: https://www.ons.gov.uk/ peoplepopulationandcommunity/wellbeing/methodologies/surveysusingthe4officefornationalstatisticspersonalwellbeingquestions (accessed on 3 February 2021).

22. Eurostat. Quality of Life: Facts and Views; Publications Office of the European Union: Luxembourg, 2015.

23. Association of Irish Market Research Organisations. Available online: https: / / aimro.ie/standards (accessed on 3 February 2021).

24. Cohen, J. Statistical Power Analysis for the Social Sciences, 2nd ed.; Lawrence Erlbaum Associates: Hillsdale, NJ, USA, 1988.

25. Vornholt, K.; Villotti, P.; Muschalla, B.; Bauer, J.; Colella, A.; Zijlstra, F.; Van Ruitenbeek, G.; Uitdewilligen, S.; Corbiere, M. Disability and employment-Overview and highlights. Eur. J. Work Organ. Psychol. 2018, 27, 40-55. [CrossRef]

26. Bodde, A.E.; Seo, D.C. A review of social and environmental barriers to physical activity for adults with intellectual disabilities. Disabil. Health J. 2009, 2, 57-66. [CrossRef] [PubMed]

27. Sellwood, D.; Raghavendra, P.; Jewell, P. Sexuality and intimacy for people with congenital physical and communication disabilities: Barriers and facilitators: A systematic review. Sex. Disabil. 2017, 35, 227-244. [CrossRef]

28. Chatzitheochari, S.; Platt, L. Disability differentials in educational attainment in England: Primary and secondary effects. Br. J. Sociol. 2019, 70, 502-525. [CrossRef] [PubMed]

29. Lucas, R.E. Long-term disability is associated with lasting changes in subjective well-being: Evidence from two nationally representative longitudinal studies. J. Personal. Soc. Psychol. 2007, 92, 717-730. [CrossRef] [PubMed] 